\title{
Wall Confinement Technique by Magnetic Gradient Inversion. Accelerators Combining Induction Effect and Pulsed Ionization. Applications
}

\author{
J.P. Petit And J. GefFray \\ LAMBDA Laboratory, France*
}

\begin{abstract}
We published the first description of a disk-shaped MHD aerodyne, combining pulsed ionization and induction systems. Magnetic pressure tends to push away electric discharge, in region of low magnetic field. In the seventies the efficiency of the wall confinement, due to the inversion of the magnetic pressure gradient was demonstrated. It is time now for ambitious MHD projects. We have planned experimental studies of MHD acceleration, combining pulsed ionization, wall confinement and induction system. We intend to study pulsed ionization systems based on wall micro discharges or microwaves action. We present the different features of the project.
\end{abstract}

PACS numbers: 47.65.-d, 52.30.Cv, 52.75.Di, 52.55.--s, 52.80.Pi

\section{Introduction}

MHD allows to strongly accelerate gases transformed into plasmas by several means. In 1976, we described the principle of an induction accelerator. Let us recall its working. If we fit the periphery of a disc with an equatorial solenoid powered by an alternating current, it will create an axisymmetric dipolar magnetic field in the surrounding gas. This varying magnetic field then induces circular eddy currents in the medium. Combining the instant value and direction of the current density $J$ and the magnetic field $B$, radial Lorentz forces $J \times B$ appear near the wall, alternatively centripetal and centrifugal as shown in Fig. 1c. Air at atmospheric pressure

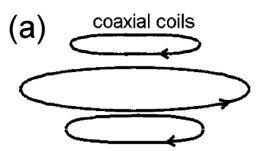

(b)

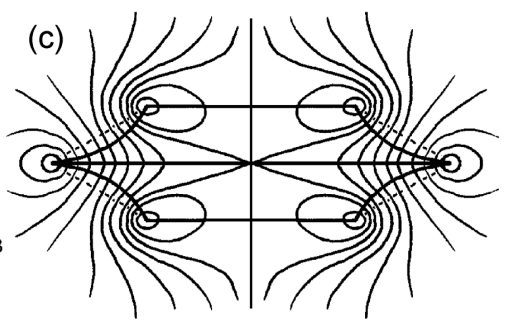

Fig. 1. (a) - coaxial coils, (b) - Lorentz force $\boldsymbol{J} \times \boldsymbol{B}$, (c) - magnetic field lines pattern.

is a good insulator, so without any ionization no force appears. Conversely, if the air is ionized everywhere all around the disc, the force field will only shake it radially in the same direction on both sides. The solution is to modulate the electrical conductivity near each side, either by covering the disc surface with little pulsed wall ionizers in Fig. 2a or by using microwaves in Fig. 2 b. The upper part is always ionized when centrifugal forces appear, the bottom using only the centripetal component. Thus the air will be sucked near the wall and a

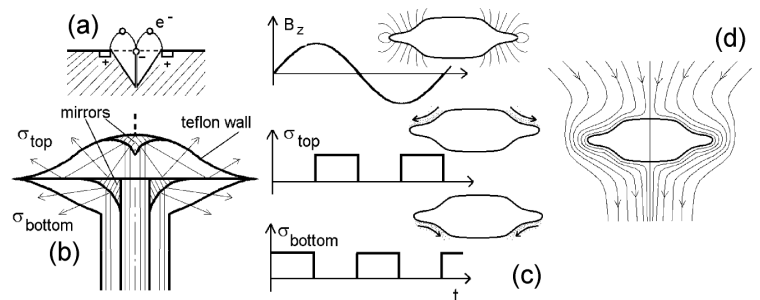

Fig. 2. Induction MHD aerodyne. (a) - wall ionizer, (b) - pulsed ionization by microwaves, (c) — varying magnetic field, ionization currents and appearing forces. (d) - induced airflow.

flow will appear in the surroundings, generating lift as seen in Fig. 2d.

\section{Experiments}

Circa 1975 we already made experiments with such external ionized airflow accelerators, which we called MHD aerodynes $[1,2]$. This formula was different, using electrodes and a high Hall effect to create spiral electric currents $[1,3,4]$. From that time, those low-pressure air experiments showed that the magnetic pressure gradient expelled the electric discharge away from the device, up to flatten it against the glass wall of the vacuum chamber. The physical explanation is simple. The electrical conductivity is

$$
\sigma=\sigma_{\mathrm{s}}\left[\begin{array}{cc}
\frac{1}{1+\beta^{2}} & \frac{-\beta}{1+\beta^{2}} \\
\frac{\beta}{1+\beta^{2}} & \frac{1}{1+\beta^{2}}
\end{array}\right],
$$

where $\beta$ is the Hall parameter and $\sigma_{\mathrm{s}}$ the scalar electrical conductivity.

The conductivity becomes very weak where the Hall parameter is the highest, in this case near the plane of symmetry. The solution is to make use of this effect, 
by reversing the magnetic gradient. This can be done by using not one, but three coaxial solenoids, as shown in Fig. 1a. The magnetic field in Fig. 1b is then maximum along a surface having the form of two truncated cones, representing the shortest path between the current loops in the solenoids. Thus the magnetic gradient effect, which proved to be effective for the cancellation of the Velikhov instability and the control of electric discharges [5], can also flatten the plasma against the wall instead of throwing it away.

We plan to develop a system combining the induction effect with the pulsed ionization near the wall. Two methods are proposed. In the first, the wall ionization is created thanks to little devices spread onto the whole surface as seen in Fig. 2a. An electric discharge establishes between the inner sting cathode and the peripheral circular anode and creates free electrons. Moreover, the magnetic field induced by such a current tends to expel these charges into the surrounding gas. The air is thus seeded with free electrons, and if the energy of these electrons is correctly chosen, they can attach to air molecules, giving negative ions with short lifespan. All these ideas lead to a range of experiments intended to test their effectiveness of such a system, which can in addition be miniaturized. The second ionization method uses two microwave beams guided into two coaxial cylindrical channels in Fig. $2 \mathrm{~b}$.

The scale model we plan to build is fitted with three solenoids as described previously, powered by three alternating currents. The hull of the device is made with Teflon, which lets the microwaves pass through it. Thanks to two mirrors, the microwaves reflect towards the walls, pass through them and ionize the air upon contact with it, near the hull. A ionized gas acts as a shield against the propagation of microwaves, and thanks to this phenomenon the plasma will be naturally created only in the immediate vicinity of the top and bottom walls. Then the two microwaves beams will be alternatively pulsed one after the other, in phase with the electric currents variations inside the solenoids, providing both propulsion and plasma confinement. In the previous paper [6] we talked about an experiment project intended to cancel the shock waves in a hot gas having a high electrical conductivity (argon at $10000 \mathrm{~K}$ in a shock tube). But the induction MHD aerodyne presented in this paper can be tested in a supersonic cold wind tunnel. We think the suction effect could be efficient enough to cancel the shock waves around the model. From this point of view, we already calculated the theoretical data [7-11]. This research field is fertile, and we plan to build other devices with electrodes.

As from 1976 we made experiments with cylindrical models as shown in Fig. 3a, described in Ref. [12]. With a $1 \mathrm{~T}$ magnetic field we made successful hydraulic analogies, in free-surface flow, of shock wave cancellation as well as turbulent wake cancellation. The device in Fig. 3b describes a spherical MHD aerodyne. Three inner coils are arranged at $120^{\circ}$ from each other and powered with alternating currents, properly dephased. Then the system creates a rotating dipolar magnetic field, in phase with a sequential power supply of pairs of diametrically opposite electrodes. The result is a flow as shown in Fig. 3b with a strong sucking effect at stagnation region.
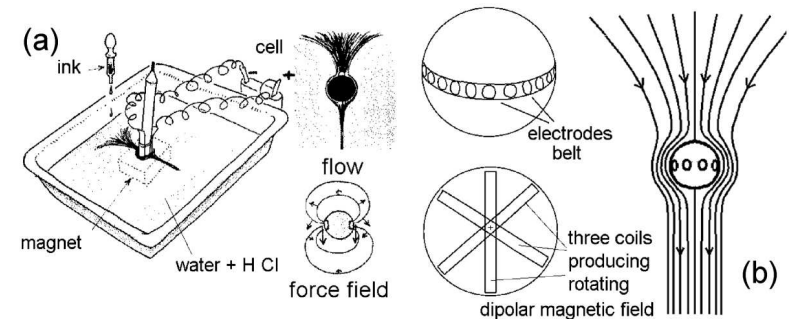

Fig. 3. (a) - 2D cylindrical MHD accelerator, "kitchen experiment", (b) - spherical MHD aerodyne, $3 \mathrm{D}$ flow.

\section{References}

[1] J.P. Petit, M. Viton, Compt. Rend. Acad. Sci. Paris 284, 167 (1977).

[2] J.P. Petit, Compt. Rend. Acad. Sci. Paris 281, 157 (1975).

[3] J.P. Petit, M. Billiotte, M. Viton, Compt. Rend. Acad. Sci. Paris 291, 129 (1980).

[4] J.P. Petit, Proc. 8th Int. Conf. on MHD Electrical Power Generation, Moscow, Russia 1983.

[5] J.P. Petit, J. Geffray, Acta Phys. Pol. A 115, 1170 (2009).

[6] J.P. Petit, J. Geffray, Acta Phys. Pol. A 115, 1149 (2009).

[7] J.P. Petit, B. Lebrun, Proc. 9th Int. Conf. on $M H D$ Electrical Power Generation, III, Part 14.E - MHD Flow, Tsukuba, Japan 1986, p. 1359.

[8] B. Lebrun, Ph.D. Thesis, Aix Marseille University, France 1987.

[9] J.P. Petit, B. Lebrun, Europ. J. Mech. B/Fluids 8, 163 (1989).

[10] J.P. Petit, B. Lebrun, Europ. J. Mech. B/Fluids 8, 307 (1989).

[11] J.P. Petit, B. Lebrun, Proc. III of 11th Int. Conf. on MHD Electrical Power Generation, Beijing, China, Part 9 - Fluid dynamics, 1992, p. 748.

[12] J.P. Petit, Proc. 8th Int. Conf. on MHD Electrical Power Generation, Moscow, Russia 1983. 